\title{
PD-L1: a promising target for TGCTs
}

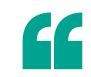

...PD-L1 expression

was higher

in patients

with more

advanced

disease...
Findings from a new study indicate that the pathway involving programmed cell death protein 1 (PD-1) and its ligand PD-L1 might offer a novel therapeutic target in testicular germ cell tumours (TGCTs).

Although TGCTs are among the most chemosensitive tumours, some patients either do not respond to cisplatin-based chemotherapy (the standard therapy), or they relapse after treatment. Inhibition of the PD-1/PD-L1 pathway is showing promise for the treatment of a number of different cancers, including melanoma, renal cell cancer and ovarian cancer. Recent data have shown that PD-L1 is overexpressed in TGCTs and not expressed in normal testicular tissue, suggesting potential for PD-1/PD-L1 as a therapeutic target in TGCTs. Cierna and colleagues aimed to evaluate the prognostic value of PD-1 and PD-L1 expression in TGCTs and associations with tumour characteristics.

The researchers used immunohistochemical techniques to evaluate PD-1 and PD-L1 expression in surgical specimens from 140 patients with TGCTs (31 pure seminomas, 70 nonseminomas and 39 mixed germ cell tumours). Expression of these proteins in tumour specimens was compared with expression in normal testicular tissue and findings were correlated with clinicopathological characteristics and clinical outcomes.

Cierna et al. found that PD-1 was not expressed in any of the TGCT samples, but that PD-L1 expression was significantly higher in TGCTs than in normal testicular tissue. PD-L1 was expressed in $76 \%$ of seminomas and $89 \%$ of nonseminomas. The researchers also found that PD-L1 expression was higher in patients with more advanced disease (for example, those with $\geq 3$ metastatic sites, increased serum tumour markers and/or nonpulmonary visceral metastases). Progression-free and overall survival were better in patients with low PD-L1 expression than in those with high PD-L1 expression.

"In this translational study, we showed for the first time prognostic value of PD-L1 expression in TGCTs and our data imply that [the] PD-1/PD-L1 pathway could be a novel therapeutic target in TGCTs," say the authors.

RebeccaKelsey

ORIGINAL ARTICLE Cierna, Z. et al. Prognostic value of programmed-death-1 receptor (PD-1) and its ligand 1 (PD-L1) in testicular germ cell tumors.

Ann. Oncol. http://dx.doi.org/10.1093/annonc/ $\underline{\mathrm{mdv} 574}$ 